\title{
Aproximación al Análisis de la Optimización de los Servicios Públicos para la Búsqueda de Empleo en una Muestra de Jóvenes
}

\author{
Sara Rodríguez Sánchez* \\ Elena Ortega Campos*** \\ Yolanda Navarro Abal ****
}

RESUMEN:

La incorporación al mundo laboral, es una de las tareas de desarrollo más relevantes y complejas, dadas las caracteristicas de la sociedad actual, para poder acceder al estatus de adulto. Con este estudio se pretende obtener información, acerca del conocimiento que los jóvenes tienen sobre las diferentes funciones del Servicio Andaluz de Empleo, la Oficina Virtual del Servicio Andaluz de Empleo $y$ del Servicio de Orientación, Información, Prácticas, Empleo y Autoempleo. Para llevar a cabo este objetivo se ha tomado una muestra de estudiantes de la Universidad de Huelva, que durante el año académico 2011/2012, se encuentran cursando su segunda titulación universitaria. La muestra está formada por 72 alumnos, de los cuales el 16.7\% son hombres y el 83\% son mujeres. La media de edad es de 23.64 años. Los resultados informan de una falta de formación e información relacionada con los Servicios Públicos de Empleo.

Palabras clave:

Adolescente, Empleo, Servicios Públicos de Empleo.

* Profesor Contratado Doctor. Departamento de Psicología Evolutiva y de la Educación. U. Huelva • sara@uhu.es

** Profesora Sustituta Interina. Departamento de Psicología Clínica, Experimental y Social. U. Huelva • elena.ortega@dpces.uhu.es

*** Profesor Cotratado Doctor. Departamento de Psicología Clínica, Experimental y Social. U. Huelva • yolanda.navarro@dpsi.uhu.es 
ABSTRACT:

The incorporation into the world of work, is one of the most relevant and complex development, by the characteristics of today's society, to gain access to adult status. This study aims to learn about the knowledge that young people have on the different functions of the Andalusian Employment Service, the Virtual Office of the Andalusian Service Employment and Guidance Services, Information, Practices, Employment and Self-Employment.

To perform this study has selected a sample of students from the University of Huelva, than during the academic year 2011/2012, are pursuing their second university degree. The sample consists of 72 students, of whom the $16.7 \%$ are men and $83 \%$ are women. The median age is 23.64 years. Results reported a lack of training and information related to the public employment services.

KEYWORDS:

Teenage, Employment, Public Employment Services.

\section{INTRODUCCIÓN}

La adolescencia, entendida como período de preparación para asumir en un futuro próximo los roles adultos, cada vez se prolonga más en el tiempo. De ahí la contradicción existente entre los planteamientos teóricos vigentes, en las teorías psicológicas del desarrollo, y la realidad actual. Muchas personas catalogadas socialmente como adolescentes, por no tener cubiertas las tareas de desarrollo exigidas para pertenecer al estatus de adulto, sobrepasan los intervalos de edad propuestos para esta primera etapa. Así, la mayoría de los estudiantes universitarios de más de 21 años se consideran y autodefinen como jóvenes y, si bien es cierto que, el intervalo de la adultez temprana tiene este origen cronológico atendiendo a la clasificación de las etapas del desarrollo de Papalia y Olds (2005), socialmente son aún catalogados como adolescentes ya que no cumplen con los mínimos exigidos socialmente para ser adultos. Esta controversia resulta de más fácil comprensión si entendemos que la adolescencia es un fenómeno cultural, no universal y que surge como consecuencia de los hechos acaecidos sobre todo a partir de la revolución industrial y la importancia que, desde entonces, se le otorga a la formación y capacitación para el trabajo. Estas circunstancias tuvieron como consecuencia la prolongación en la preparación y formación para el empleo, alargando los años de estudios y en consecuencia el inicio de las tareas de desarrollo propias de los adultos. En este sentido, la adolescencia puede definirse como compás de espera que la sociedad otorga a sus miembros más jóvenes mientras se preparan para asumir los roles adultos (Erikson, 1993). 
Una de las tareas de desarrollo que la sociedad impone a sus miembros para poder acceder al estatus de adulto es la independencia económica que, en circunstancias normales, sólo es posible mediante la ocupación de un puesto de trabajo. La integración en el mercado laboral constituye uno de los roles principales a desarrollar por los jóvenes adultos (Nelson, 1990). El trabajo es considerado una fuente de ingreso que facilita la historia de vida y genera aportaciones psicosociales de gran relevancia para el desarrollo integral de las personas. Las funciones psicosociales que pueden atribuirse al trabajo son muchas. Algunos autores como Salanova, Gracia y Peiró (1996) han señalado como más relevante las siguientes:

1. Función integradora: Favorece el desarrollo de la identidad laboral y personal.

2. Función de proporcionar estatus y prestigio social.

3. Función de conceder identidad personal.

4. Función económica.

5. Función como fuente de oportunidades para la interacción y las redes sociales.

6. Función de estructuración del tiempo.

7. Función de mantener al individuo bajo una actividad más o menos obligada.

8. Función de ser una fuente de oportunidades para desarrollar habilidades o destrezas.

9. Función de trasmitir normas, creencias y expectativas sociales.

10. Función de proporcionar control y poder.

11. Función de comodidad.

Actualmente nuestros adolescentes viven una situación ciertamente paradójica en el área laboral, desde la perspectiva de que cada vez se les exige una mayor preparación para ocupar un puesto de trabajo y, sin embargo, las oportunidades para poder acceder al mundo laboral son cada vez más inseguras e inciertas. Por otro lado, las ofertas de empleo para los jóvenes, están caracterizadas en su mayoría por tener importantes niveles de precariedad y continuidad en el tiempo. En este sentido, el Ministerio de Trabajo y Asuntos Sociales, y más específicamente el Observatorio de la Juventud en España, publicó los resultados de una encuesta en la que el $77 \%$ de los jóvenes encuestados consideraban que la seguridad y la estabilidad eran las características más relevantes que debe tener un trabajo. Algunos autores como Cepal (2004), consideran que un factor clave para el 
presente y el futuro de los adolescentes y de los jóvenes es la manera en que ambos configuran su inserción laboral.

Con la finalidad, entre otras, de favorecer una mayor empleabilidad y en consecuencia la inserción laboral de los jóvenes, se crea el Servicio Andaluz de Empleo (SAE) según aparece recogido en la Ley 4/2002, de 16 de diciembre (BOJA, n . 153, 2002). En ella, en el Capítulo I sobre Naturaleza y Atribuciones, y según su Artículo 3 que refiere las Funciones del Servicio Andaluz de Empleo, se recogen entre ellas: por un lado, la intermediación laboral, el registro de demandantes de empleo, la recepción de comunicación de contratos y la gestión de la red Eures en Andalucía y, por otro, la orientación e información profesional, y las acciones de apoyo para la mejora de la cualificación profesional y el empleo.

En las últimas décadas el uso de internet ha adquirido una relevancia de tanta magnitud, que ha constituido en muchas ocasiones un serio problema el consumo excesivo del mismo. Así, algunos autores (Estallo, 2001; Echeburúa, Labrador, y Becoña, 2009; Estévez, Bayón, De la Cruz y Fernández-Liria, 2009), informan que el internet es una de las principales adicciones comportamentales de los últimos años. No obstante, no ajenos a la gran oportunidad que ofrece como herramienta de difusión, formación, socialización, etc. y otras diferentes funciones que favorecen las políticas activas de empleo y, por tanto, la mejora de la empleabilidad en el ciudadano, el Servicio Andaluz de Empleo, y según el artículo 13 del Estatuto de Autonomía, tuvo también como objetivo para su creación potenciar la capacidad de generar empleo $y$ riqueza en nuestra sociedad, con el condicionante de ir permitiendo adecuar el mercado de trabajo al profundo proceso de cambio tecnológico que se viene produciendo.

Posteriormente, y siguiendo las directrices de los Servicios Públicos de Empleo, las instituciones de la administración pública, y más específicamente las instituciones educativas, se hicieron eco y crearon sus propios servicios para dar respuesta a las demandas de sus usuarios. De esta forma, entre los servicios que ofrece la Universidad de Huelva para los estudiantes se encuentra el Servicio de Orientación e Información, Prácticas, Empleo y Autoempleo (SOIPEA) que a lo largo de todo el curso académico ofrece al alumnado Servicios de Orientación Profesional, Prácticas en empresa e instituciones, asesoramiento para el autoempleo y emprendimiento, acciones formativas, una bolsa de empleo para los que hayan finalizado sus estudios y acciones específicas para la mejora de la empleabilidad de la mujer universitaria. Una de las peculiaridades de este servicio es la gran difusión y dinamismo que ofrece a lo largo de todo el curso, destacando la gran visibilidad en tablones de anuncio, pantallas flash, jornadas, etc.

Por tanto, resulta curioso que cuando entre el alumnado se realiza la técnica del torbellino de ideas con el objetivo de obtener información sobre el conocimiento que poseen acerca de los Servicios Públicos de Empleo y sus recursos en la red, se 
aprecia un desconocimiento, o al menos parcial, acerca del mismo. Esta prestación de los Servicios Públicos a pesar de ser un recurso de gran relevancia y apoyo para la consecución de un posible empleo, parece no ser excesivamente visible entre los adolescentes como entidad física.

Estos datos no llevan a la reflexión y planteamiento de diferentes cuestiones que se exponen a continuación relacionadas, todas ellas, con la carencia informativa de los adolescentes en relación a los recursos que ofrece el Servicio Público de Empleo para una óptima transición para al mercado laboral:

1. Una carencia de importancia es la centralización en el trabajo, centralizando sus vidas en la búsqueda de otras alternativas. Es decir, entre los valores prioritarios de los adolescentes no está contemplado, aquél cuyo núcleo básico es el laboral.

2. Una falta de difusión y visibilidad de los Servicios de la Administración Pública de Empleo.

3. La creencia estereotipada de las funciones de los Servicios Públicos de Empleo quedan reducidas a lo que el alumnado conoce como "la tarjeta del paro".

4. Las bajas expectativas del alumnado con respecto a las oportunidades del mercado laboral.

5. El hecho de tener cubierta sus principales necesidades, les lleva a despreocuparse de otras actividades que están orientadas hacia su autorrealización profesional.

6. Falta de empoderamiento (empowerment) en la búsqueda de sus propios recursos.

A partir de estas reflexiones, surge el objetivo del presente estudio por la necesidad de tomar conciencia del grado de conocimiento que los jóvenes tienen sobre las diferentes funciones del Servicio Andaluz de Empleo, la oficina virtual del SAE y del SOIPEA, mucho más accesible y orientado a la población universitaria de Huelva.

\section{MÉTODO}

El estudio se ha realizado con una muestra de estudiantes de la Licenciatura de Psicopedagogía, durante el curso académico 2011/2012. En la mayoría de los casos han realizado previamente la Diplomatura de Educación Social o la Diplomatura de Magisterio). Los alumnos, voluntariamente, contestaron a una serie de 
preguntas sobre su conocimiento sobre el SAE, mediante un protocolo de recogida de información, con una duración de aproximadamente 15 minutos.

La muestra de este estudio está formada por 72 alumnos, según el sexo, el $16.7 \%(\mathrm{~N}=12)$ son hombres y el $83.3 \%(\mathrm{~N}=60)$ son mujeres. El rango de edad de la muestra se sitúa entre 22 y 44 años, solamente 4 personas sobrepasan los 30 años. La media de edad de la muestra toma un valor de 23.64 años y la mediana toma un valor de 22.50 años.

Según los estudios previos, el 18.1\% $(\mathrm{N}=13)$ ha realizado la Titulación de Educación Social, el 12.5\% ( $\mathrm{N}=9)$ Maestro en Educación Primaria, el 26.4\% (N = 19) Maestro en Educación Infantil, el 22.2\% ( $\mathrm{N}=16)$ Maestro de Educación Física, el 2.8\% ( $\mathrm{N}=2)$ Maestro en Educación Musical, el 2.8\% ( $\mathrm{N}=2)$ Maestro de Lengua Extranjera y en el $8.3 \%$ de los casos $(\mathrm{N}=6)$ se desconoce los estudios previos que han realizado. Según el año en que finalizaron la diplomatura que da acceso a la Licenciatura en Psicopedagogía, el 4.2\% ( $\mathrm{N}=3)$ finalizó sus estudios en 2005, el 2.8\% ( $\mathrm{N}=2)$ en 2007, el 6.9\% ( $\mathrm{N}=5)$ en 2008, el 5.6\% ( $\mathrm{N}=4)$ en 2009 , el $22.2 \%(\mathrm{~N}=16)$ en 2010 , el $51.4 \%(\mathrm{~N}=37)$ en 2011 y en el $6.9 \%(\mathrm{~N}=$ 5) de los casos se desconoce el año de finalización de los estudios que les han dado acceso a la Licenciatura.

Respecto a la experiencia laboral previa de los participantes de este estudio, el $37.5 \%(\mathrm{~N}=27)$ ha trabajado desde que finalizó sus estudios superiores, mientras que el 62.5\% ( $\mathrm{N}=45)$ no han trabajado desde que han finalizado sus estudios superiores. En la situación laboral presente de los participantes, el 18.1\% $(\mathrm{N}=13)$ trabaja en la actualidad, por el contrario, el $81.9 \%(\mathrm{~N}=59)$ no trabaja actualmente (ver tabla 1 ).

Tabla 1- Descripción de la muestra

\begin{tabular}{|c|c|c|c|}
\hline Variable & Valores & Frecuencia & Porcentaje \\
\hline \multirow[t]{2}{*}{ Sexo } & Hombre & 12 & 16.7 \\
\hline & Mujer & 60 & 83.3 \\
\hline \multirow[t]{8}{*}{ Estudios realizados } & Educación social & 13 & 18.1 \\
\hline & Magisterio primaria & 9 & 12.5 \\
\hline & Magisterio infantil & 19 & 26.4 \\
\hline & Magisterio Ed. Especial & 16 & 22.2 \\
\hline & Magisterio Ed. Física & 5 & 6.9 \\
\hline & Magisterio Musical & 2 & 2.8 \\
\hline & Magisterio Lengua Extranjera & & 2.8 \\
\hline & Se desconoce & 6 & 8.3 \\
\hline
\end{tabular}




\begin{tabular}{|c|c|c|c|}
\hline Año fin estudios & 2005 & 3 & 4.2 \\
\hline & 2007 & 2 & 2.8 \\
\hline & 2008 & 5 & 6.9 \\
\hline & 2009 & 4 & 5.6 \\
\hline & 2010 & 16 & 22.2 \\
\hline & 2011 & 37 & 93.1 \\
\hline & Se desconoce & 5 & 6.9 \\
\hline \multirow[t]{2}{*}{$\begin{array}{l}\text { ¿Has trabajado desde que finalizaste los } \\
\text { estudios? }\end{array}$} & $\mathrm{Si}$ & 27 & 37.5 \\
\hline & No & 45 & 62.5 \\
\hline \multirow[t]{2}{*}{ ¿Trabajas actualmente? } & $\mathrm{Si}$ & 13 & 18.1 \\
\hline & No & 59 & 81.9 \\
\hline
\end{tabular}

RESULTADOS

Conocimiento del SOIPEA (Servicio de Orientación, Información, Prácticas, Empleo y Autoempleo) de la Universidad de Huelva.

Ante la pregunta acerca de si conocen el SOIPEA de la Universidad de Huelva, el 41.7\% ( N = 30) de la muestra, dice conocer el Servicio de Orientación, Información, Prácticas, Empleo y Autoempleo de la Universidad de Huelva, por el contrario, el 58.3\% ( $\mathrm{N}=42)$ de la muestra no conoce dicho servicio de la universidad. Del total de alumnos que dicen conocer el SOIPEA, se quiso obtener información sobre qué canal se produjo dicho conocimiento. El 22.2\% $(\mathrm{N}=16)$ dice haberlo conocido a través de publicidad hecha por el SOIPEA, el 13.9\% $(\mathrm{N}=10)$ por medio de los compañeros de clase, el 2.8\% ( $\mathrm{N}=2)$ mediante profesores de la universidad, el $1.4 \%(\mathrm{~N}=1)$ por su familia y, por último, el 1.4\% $(\mathrm{n}=1)$ no aporta dicha información. Del total de alumnos que conocían el SOIPEA $(\mathrm{N}=30)$, queríamos conocer cuántos de ellos habían acudido al servicio. El 53.3\% ( $\mathrm{N}=16)$ había acudido al SOIPEA, mientras el 46.7\% ( $\mathrm{N}=14$ ) no había acudido. De los alumnos que había acudido al SOIPEA, el 37.5\% ( $\mathrm{N}=6$ ) habían asistido motivados por conseguir "prácticas para estudiantes", el 18.8\% $(\mathrm{N}=3)$ para conseguir "prácticas para titulados", el 6.3\% $(\mathrm{N}=1)$ para inscribirse en la bolsa de empleo que tiene dicho servicio, el $12.5 \%(\mathrm{~N}=2)$ para recibir orientación profesional, el 2.8\% ( $\mathrm{N}=2)$ para conseguir formación para el empleo, y, por último, el 2.8\% ( $\mathrm{N}=2)$ manifestaba más de un motivo (prácticas para estudiantes, prácticas para titulados, bolsa de empleo y orientación profesional).

Asimismo, se quiso conocer si la visita al SOIPEA les había ayudado en la búsqueda de empleo, del total de estudiantes $(\mathrm{N}=16)$ que habían acudido voluntariamente al servicio SOIPEA, el 50\% $(\mathrm{N}=8)$ dice que le han ayudado en la búsqueda de em- 
pleo, mientras el otro 50\% ( $\mathrm{N}=8)$ manifiesta lo contrario. También se le preguntó a los alumnos, si el SOIPEA les había ayudado a encontrar empleo, del total de alumnos que habían acudido al SOIPEA $(\mathrm{N}=16)$, el 18.8\% $(\mathrm{N}=3)$ dice que el servicio le ha ayudado a encontrar empleo, por el contrario, el $81.3 \%(\mathrm{~N}=13)$ expresa lo contrario (ver tabla 2).

TABla 2. Conocimiento Del SOIPEA

\begin{tabular}{|c|c|c|c|}
\hline Variable & Valores & Frecuencia & Porcentaje \\
\hline \multirow[t]{2}{*}{ ¿Conoces el SOIPEA? } & $\mathrm{Si}$ & 30 & 41.7 \\
\hline & No & 42 & 58.3 \\
\hline \multirow[t]{5}{*}{ ¿Cómo conociste el SOIPEA? } & Publicidad en la UHU & 16 & 55.2 \\
\hline & Compañeros de clase & 10 & 34.5 \\
\hline & Profesores & 2 & 6.9 \\
\hline & Familia & 1 & 3.4 \\
\hline & Se desconoce & 1 & 3.4 \\
\hline \multirow[t]{2}{*}{ ¿Has acudido al SOIPEA? } & $\mathrm{Si}$ & 16 & 53.3 \\
\hline & No & 14 & 46.7 \\
\hline \multirow[t]{6}{*}{ Motivo de la visita al SOIPEA } & Prácticas para estudiantes & 6 & 37.5 \\
\hline & Prácticas para titulados & 3 & 18.8 \\
\hline & Bolsa de empleo & 1 & 6.3 \\
\hline & Orientación profesional & 2 & 12.5 \\
\hline & Formación para el empleo & 2 & 12.5 \\
\hline & $\begin{array}{l}\text { Prácticas estudiantes, prácticas } \\
\text { titulados, bolsa empleo y orientación } \\
\text { profesional }\end{array}$ & 2 & 12.5 \\
\hline \multirow[t]{2}{*}{$\begin{array}{l}\text { SOIPEA te ha ayudado en la búsqueda } \\
\text { de empleo }\end{array}$} & $\mathrm{Si}$ & 8 & 50 \\
\hline & No & 8 & 50 \\
\hline \multirow[t]{2}{*}{$\begin{array}{l}\text { SOIPEA te ha ayudado a encontrar } \\
\text { empleo }\end{array}$} & $\mathrm{Si}$ & 3 & 18.8 \\
\hline & No & 13 & 81.3 \\
\hline
\end{tabular}




\section{Conocimiento del SAE (Servicio Andaluz de Empleo).}

En un segundo bloque, se quería conocer el conocimiento que tenían los estudiantes del SAE, del total de la muestra de este trabajo, el 63.9\% ( $\mathrm{N}=46)$ dice conocer el SAE, mientras que el 36.1\% ( $\mathrm{N}=26)$ no tiene conocimiento del mismo. Se consideró importante averiguar la vía por la cual habían tenido conocimiento del SAE, en el 7\% (N = 3) de los casos, a través de publicidad en la Universidad de Huelva, el 20.9\% ( $\mathrm{N}=9)$ por compañeros de clase, el $7 \%(\mathrm{~N}=3)$ por profesores, el 18.6\% ( $\mathrm{N}=8)$ por amigos, el $37.2 \%(\mathrm{~N}=16)$ por la familia, el $9.3 \%(\mathrm{~N}=4)$ por publicidad realizada en los medios y, por último, en el $7 \%$ de los casos $(\mathrm{N}=3)$ se desconoce como tuvieron conocimiento del SAE.

Del total de alumnos que tenían conocimiento de la existencia del SAE, se quería saber cuántos habían acudido al mismo, se encontró que un 63\% ( $\mathrm{N}=29)$ había acudido al SAE, mientras que un 37\% ( $\mathrm{N}=17)$ no lo había hecho. Los motivos por los que el alumnado acude al SAE son, el 62.1\% ( $\mathrm{N}=18)$ "buscando un empleo", el 3.4\% $(\mathrm{N}=1)$ buscando "asesoramiento para el autoempleo", el 3.4\% ( $\mathrm{N}=1)$ por "prestaciones de desempleo", el 6.9\% ( $\mathrm{N}=2)$ buscando "formación para el empleo", el 3.4\% ( $=1)$ "buscando empleo y asesoramiento para el autoempleo" y, por último, el 20.7\% ( $=6)$ "buscando empleo, prestación por desempleo y formación para el empleo".

Ante la pregunta de si el SAE le había ayudado en la búsqueda de empleo, el $40 \%(\mathrm{~N}=12)$ de los alumnos que habían acudido al SAE, dicen que les ha ayudado en la búsqueda de empleo, mientras que el 60\% ( $\mathrm{N}=18)$ dice lo contrario. Respecto si haber acudido al SAE le había ayudado a encontrar empleo, el 6.7\% ( N = 2) de los estudiantes dice que le había ayudado a encontrar trabajo, mientras que el 93.3\% (N = 28) manifiestan lo opuesto (ver tabla 3).

Tabla 3. Conocimiento del SAE

\begin{tabular}{|l|l|l|l|}
\hline Variable & Valores & Frecuencia & Porcentaje \\
\hline \multirow{2}{*}{ ¿Conoces el SAE? } & Si & 46 & 63.9 \\
\hline & No & 26 & 36.1 \\
\hline & & & \\
\hline ¿Cómo conociste el SAE? & Publicidad en la UHU & 3 & 7 \\
\hline & Compañeros de clase & 9 & 20.9 \\
\hline & Profesores & 3 & 7 \\
\hline & Amigos & 8 & 18.6 \\
\hline & Familia & 16 & 37.2 \\
\hline & Publicidad & 4 & 9.3 \\
\hline & Se desconoce & 3 & 7 \\
\hline
\end{tabular}




\begin{tabular}{|l|l|l|l|}
\hline \multicolumn{4}{|l|}{} \\
\hline ¿Has acudido al SAE? & Si & 29 & 63 \\
\hline & No & 17 & 37 \\
\hline Motivo de la visita al SAE & Búsqueda de empleo & 18 & 62.1 \\
\hline & Asesoramiento Autoempleo & 1 & 3.4 \\
\hline & Prestaciones desempleo & 1 & 3.4 \\
\hline & Formación empleo & 2 & 3.9 \\
\hline & $\begin{array}{l}\text { Búsqueda empleo y asesoramiento } \\
\text { autoempleo }\end{array}$ & 1 & 3.4 \\
\hline $\begin{array}{l}\text { SAE te ha ayudado en la búsqueda } \\
\text { de empleo }\end{array}$ & $\begin{array}{l}\text { Búsqueda de empleo, prestación } \\
\text { desempleo y formación para el empleo }\end{array}$ & 6 & 20.7 \\
\hline & Si & 12 & 40 \\
\hline $\begin{array}{l}\text { SAE te ha ayudado a encontrar } \\
\text { empleo }\end{array}$ & No & 18 & 60 \\
\hline & Si & 2 & 6.7 \\
\hline
\end{tabular}

\section{Conocimiento de la oficina virtual del SAE (Servicio Andaluz de Empleo)}

Un último bloque, hace referencia al conocimiento que tenían los alumnos de la oficina virtual del SAE, el 58.3\% ( $\mathrm{N}=42)$ dicen tener conocimiento de la existencia de la oficina virtual, mientras que el $41.7 \%(\mathrm{~N}=30)$ desconoce que exista. A los alumnos que tenían conocimiento de la oficina virtual, se les preguntaba si habían hecho uso de ella, un 54.35\% ( $\mathrm{N}=24)$ dice haber utilizado la página web, mientras que el 45.65\% ( $\mathrm{N}=21)$, a pesar de conocerla, no ha hecho uso de ella. Asimismo, se quiso saber si a los alumnos que conocían dicha oficina virtual, les parecía útil en el proceso de búsqueda de empleo, un $69.4 \%(\mathrm{~N}=25)$ la consideró útil proceso de búsqueda de empleo, mientras que un 30.6\% $(\mathrm{N}=11)$ manifestó lo contrario. Por último, se preguntó qué acciones habían realizado a través de la oficina virtual del SAE, un $11.1 \%(\mathrm{~N}=3)$ había solicitado cita en Andalucía Orienta, un 29.6\% ( $\mathrm{N}=8)$ había renovado la tarjeta de solicitud de empleo, un $18.5 \%(\mathrm{~N}=5)$ había solicitado cursos de formación para el empleo, un 3.7\% $(\mathrm{N}=1)$ la había utilizado para buscar empleo, un $7.4 \%(\mathrm{~N}=2)$ para pedir cita en Andalucía Orienta y renovar la tarjeta de empleo, por último, un 29.6\% ( $\mathrm{N}=8)$ para pedir cita en Andalucía Orienta, renovar la tarjeta de empleo y solicitar cursos de formación para el empleo (ver tabla 4). 
Tabla 4. Conocimiento de la oficina virtual del SAE

\begin{tabular}{|c|c|c|c|}
\hline Variable & Valores & Frecuencia & Porcentaje \\
\hline \multirow[t]{2}{*}{ ¿Conoces la oficina virtual del SAE? } & $\mathrm{Si}$ & 42 & 58.3 \\
\hline & No & 30 & 41.7 \\
\hline \multirow{2}{*}{$\begin{array}{l}\text { ¿Has utilizado la oficina virtual del } \\
\text { SAE? }\end{array}$} & Si & 25 & \\
\hline & No & 21 & \\
\hline \multirow[t]{2}{*}{$\begin{array}{l}\text { ¿Crees que la oficina virtual del SAE es } \\
\text { útil en la búsqueda de empleo }\end{array}$} & Si & 25 & 69.4 \\
\hline & No & 11 & 30.6 \\
\hline \multirow[t]{6}{*}{$\begin{array}{l}\text { Acciones que has realizado a través de } \\
\text { la oficina virtual del SAE }\end{array}$} & Solicitud cita Andalucía Orienta & 3 & 11.1 \\
\hline & Renovar tarjeta empleo & 8 & 29.6 \\
\hline & Solicitar cursos FPO & 5 & 18.5 \\
\hline & Buscar empleo & 1 & 3.7 \\
\hline & $\begin{array}{l}\text { Cita Andalucía Orienta y Renovar } \\
\text { tarjeta empleo }\end{array}$ & 2 & 7.4 \\
\hline & $\begin{array}{l}\text { Cita Andalucía Orienta, Renovar } \\
\text { tarjeta empleo y solicitar cursos FPO }\end{array}$ & 8 & 29.6 \\
\hline
\end{tabular}

\section{DISCUSIÓN}

Conocimiento del SOIPEA (Servicio de Orientación, Información, Prácticas, Empleo y Autoempleo) de la Universidad de Huelva.

En este apartado, se reflexiona sobre los datos obtenidos que más interés han despertado en el estudio realizado. Con respecto al número de alumnos que dicen conocer el SOIPEA, llama la atención cómo de los 72 estudiantes sólo 30 de ellos conocían este servicio. Ante la pregunta acerca de si conocen el SOIPEA de la Universidad de Huelva, el 41.7\% ( $\mathrm{N}=30)$ de la muestra, dice conocer el Servicio de Orientación, Información, Prácticas, Empleo y Autoempleo de la Universidad de Huelva, por el contrario, el 58.3\% ( $\mathrm{N}=42)$ no lo conocen.

De los datos obtenidos, con respecto a la variable por qué canal se produjo el conocimiento del servicio, llama la atención que sólo el 2,8\% dice haberlo conocido 
por los profesores. En los últimos años, se está viviendo un cambio educacional fruto de la implantación del Espacio Europeo de Educación Superior. Así, tal como manifiestan Climent y Navarro (2009), en España, el Real Decreto de 29 de octubre de 2007, en el que se establece la ordenación de las enseñanzas oficiales, constituye el punto de inflexión de la adaptación de nuestro sistema de enseñanza superior. En él se señala como base de la reforma, la importancia de las competencias profesionales y de la empleabilidad. También, se resalta la adquisición de competencias profesionales como objetivo prioritario en los estudios universitarios, sin excluir la necesidad de los contenidos y las horas lectivas necesarias para la adquisición de otras competencias y conocimientos. El profesorado debe ser consciente del cambio educacional acaecido y tomar más protagonismo en el desempeño de otras funciones diferentes a la simple exposición de contenidos de aprendizaje. En cualquier caso, sería de interés de otra investigación abordar los conocimientos que el profesorado posee sobre estos sistemas ya que, quizás, sería necesario inculcar y sensibilizar sobre la necesidad de conocer dichos recursos para asesorar y fomentar el buen uso de los mismos, favoreciendo el aumento de oportunidades en el mercado de trabajo de nuestros estudiantes.

Resulta interesante que sólo la mitad de alumnos que conocen el servicio han acudido al SOIPEA. En este sentido, si se presta atención al porcentaje acumulado, se observa como un elevado número de alumnos han hecho uso de este servicio para buscar prácticas y formación. El motivo de asistencia al servicio hace recapacitar sobre la formación aplicada que reciben los alumnos, llamando la atención que una necesidad que manifiestan es la búsqueda de formación y prácticas. Este dato lleva a diferentes explicaciones: por un lado, la evidencia de que la formación de Grado es una enseñanza que a posteriori requiere otra complementaria que permita la especialización y profundización de los conocimientos. No obstante, se debe cuestionar, si la enseñanza que se ofrece está dotada de suficiente transferencia del conocimiento y se ajusta a las necesidades que la oferta del mercado laboral demanda; por otro lado, otra interpretación que se puede llevar a cabo viene al hilo de los planteamientos que surgieron para realizar este trabajo. Se hace referencia a la poca importancia del trabajo como valor centralizado en la vida de los jóvenes. Un dato curioso teniendo en cuenta que este alumnado ya posee una titulación, es que sólo uno de ellos ha acudido para ser inscrito en la bolsa de empleo. Este hecho se intensifica cuando los mensajes masivos que se refieren a la sociedad actual, hablan de "sociedad del conocimiento". Esta expresión lleva implícita que el alumnado escuche a lo largo de todo el proceso de enseñanza-aprendizaje varios mensajes que le pueden llevar a error en la conformación de su currrículum profesional. Entre ellos se encuentra, la necesidad de formación, de realizar prácticas que le ayudarán a desarrollar competencias profesionales y la interdisciplinariedad o la importancia de las competencias transversales, básico todos ellos para el aumento de la empleabilidad. En muchos casos, este mensaje hace que el alumnado no consiga generar un buen itinerario 
personalizado en su currículum intentando optar a cualquier formación que le permita un acumulo de certificaciones pero que, habitualmente, no le otorgan un perfil idóneo. Cuando acuden a los servicios de orientación, los usuarios han coleccionado certificaciones, entendiendo que "un currículum al peso" es un currículum de calidad. Se hace evidente, por tanto, desde el rol docente universitario, debatir y plantear un nuevo sistema educativo que requiera de la cualificación del profesorado para informar y asesorar sobre los servicios disponibles como indispensables a lo largo de la vida laboral de la persona y, a su vez, plantear si verdaderamente la enseñanza realizada es una enseñanza aplicada o una enseñanza desde los modelos teóricos. Y, en el caso de esta última opción, si verdaderamente no sería contradictorio con el nuevo sistema de enseñanza superior.

El siguiente apartado que sería de interés analizar es el relacionado con la percepción de ayuda que tienen las personas que acudieron al servicio. Es evidente que tanto en lo referido a la búsqueda como al hecho de haberles ayudado a encontrar empleo el porcentaje encontrado es muy pequeño. Quizás este apartado se podría ampliar con la información obtenida en los debates grupales que se originan en las clases. Sería de interés definir qué concepto tienen sobre "ofrecer ayuda". En esta ocasión, se debe mencionar otra de las causas que llevó a la realización de este trabajo. Así, nuevamente se encuentra con otra paradoja de esta sociedad actual. En este sentido, y sobre todo, inicialmente desde los estudios de las organizaciones, se comenzó a hablar ya hace varios años de la relevancia del empoderamiento como una herramienta que otorga poder a la persona. Paradójicamente, comienzan a aparecer nuevas figuras que asesoran, orientan, etc. a las personas en sus diferentes ámbitos de la vida y períodos del ciclo vital. Ejemplo de ellos lo encontramos en los orientadores educativos, orientadores laborales, coach, mediadores, etc. Esta contradicción, y volviendo a la temática de estudio, lleva a la reflexión de pensar si, realmente, los nuevos alumnos están desarrollando competencias para el desempeño autónomo de su búsqueda de empleo o, por el contrario, estamos haciendo personas dependientes que requieran siempre de otras para poder tomar decisiones en aquellas áreas de la vida que van a determinar su historia personal. De esta manera, es posible que el alumnado entienda como ayudar a buscary a encontrar empleo una función diferente a la que el recurso del SOIPEA les pueda mostrar.

\section{Conocimiento del SAE (Sistema Andaluz de Empleo) y la oficina virtual de empleo}

Del total de la muestra, el 63.9\% dice conocer el SAE y llama la atención como esta información proviene básicamente de la familia (37.2\%) y, seguidamente los compañeros de clase (20.9\%). Es necesario detenerse en este dato ya que, a pesar de ser un colectivo, que posee todas las herramientas formativas e informativas para acceder al mercado laboral, sin embargo, la información no es obtenida de esos 
recursos. En este país existe una cultura del empleo basada en finalizar la formación e inscribirse en la oficina de empleo esperando que "te llamen para trabajar". Con el paso de los meses, sin que este hecho ocurra, se atribuyen deficiencias de funciones y carencias en la utilidad de los servicios que se ofrecen desde el SAE. Esta cultura, se trasladará con posterioridad a los servicios de orientación, en donde el usuario, considera que el profesional de la orientación debe ofrecerle "una carta de ofertas de empleo" para que la persona pueda acceder sintiéndose defraudado cuando esto no sucede. Como se ha comentado en el apartado anterior una de las peculiaridades observadas en el alumnado es su falta de empoderamiento. En este sentido, se podría resaltar la falta de iniciativa, toma de decisiones y capacidad de emprendimiento que se observan en todas las áreas de desarrollo vital, y muy específicamente en el tema de estudio, es decir, en el ámbito laboral. Cuando finalizan los estudios esperan como primer recurso para la búsqueda de empleo "la red de contactos" que les pueda ofrecer sus padres, madres, etc. y, sobre todo, en estos momentos (donde la razón les acompaña), atribuyen la falta de empleo a la situación de crisis así como a que se encuentran desmotivados y piensan que su frustración es la causa de no insistir y cesar en la búsqueda de empleo. Por tanto, sería de especial interés dar a conocer realmente las posibilidades que ofrece el SAE para que las expectativas de los usuarios sean más realistas y comprenden cual es su responsabilidad dentro del proceso de la incorporación al mercado laboral.

Así, como los datos indican, es significativo observar como el $62.1 \%$ de los alumnos acuden al SAE con el objetivo de buscar empleo. Las principales causas que los jóvenes informan por las que acuden al servicio de empleo son la demanda de empleo (en lenguaje de la calle "sacarme la tarjeta del paro"); renovación de la demanda de desempleo (en el lenguaje coloquial "sellar el paro"); y, la búsqueda de cursos de formación gratuitos y, especialmente, que tengan compromiso de contratación. Nuevamente se observa la falta de conocimiento de los servicios públicos que tienen al alcance así como la falta de responsabilidad manifestada en la pasividad que muestran en su propio proceso de búsqueda de empleo.

Para finalizar, y en relación al conocimiento de la oficina virtual del SAE, los resultados obtenidos siguen en la misma línea que los anteriores. Resulta de interés observar como los alumnos denominados nativos tecnológicos por pertenecer a la generación 2.0 y, como se ha referido anteriormente, incluso llegando a padecer patologías emergentes debido al uso y abuso de las nuevas tecnologías, queda demostrado que a pesar de los conocimientos que tienen de las nuevas tecnologías y de manifestar que pueden pasar horas vagabundeando por la red, sin embargo, esta página no despierta interés, no siendo capaces de usarla ni sentirla como un beneficio propio y mostrando una falta de interés en su proceso de búsqueda de empleo. 


\section{BIBLIOGRAFÍA}

BOLETÍN OFICIAL DE LA JUNTA DE ANDALUCÍA (2002): Ley 4/2002, de 16 de diciembre, de creación del Servicio Andaluz de Empleo (n ${ }^{\circ} 153$, pp. 24995-24999).

CLIMENT Y NAVARRO (2010): "Importancia de la orientación profesional en el desempeño de competencias profesionales y aumento de la empleabilidad dentro del marco del espacio europeo de educación superior", Revista Qurriculum, 23, pp. 165-177.

ERIKSON, E. H. (1993): Sociedad y adolescencia, México, Siglo XXI.

ESTALlO, J.A. (2001): Usos y abusos de Internet, Anuario de Psicología, 32, pp. 95-108.

ESTÉVEZ, L., BAYÓN, C., DE LA CRUZ, J. y FERNÁNDEZ-LIRIA, A. (2009): “Uso y abuso de Internet en adolescentes". En E. Echeburúa, F.J. Labrador y E. Becoña (eds.), Adicción a las nuevas tecnologías en adolescentes y jóvenes, Madrid, Pirámide, pp. 101-128.

ECHEBURÚA, E., LABRADOR, F.J. y BECOÑA, E. (2009): Adicción a las nuevas tecnologías en adolescentes jóvenes, Madrid, Pirámide.

NELSON, D.L. (1990): "Adjusting to a new organization: easing the transition from outsider to insider", Prevention in Human Services, 8 (1), pp. 61-86.

PAPALIA, D. Y OLDS, S.W. (2005): Desarrollo bumano, México, McGraw-Hill.

SALANOVA, M., GRACIA, F.J. y PEIRÓ, J.M. (1996): "Significado del trabajo y valores laborales". En J.M. Peiró y F. Prieto (eds.), Tratado de Psicología del Trabajo. Vol. II: Aspectos psicosociales del trabajo, Madrid, Síntesis, pp. 35-64. 